\title{
Prevalência de cistos ovarianos em vacas de alta produção submetidas a sistema de confinamento e dieta total
}

\author{
Prevalence of ovarian cysts in production high cows under containment system and diet \\ total
}

\author{
Rodrigo Garcia Motta ${ }^{1}$, Lorrayne de Souza Araújo Martins ${ }^{1 *}$, Gustavo Silva Medeiros ${ }^{2}$ e Valdir \\ Chiogna Junior ${ }^{2}$
}

${ }^{1}$ Universidade Estadual Paulista, Botucatu, SP, Brasil. *Autor para correspondência: lorraynevet@hotmail.com.

${ }^{2}$ Médico Veterinário Autônomo, Rio Verde, GO, Brasil.

Submissão:12/09/2018 / Aceite: 01/07/2019

\begin{abstract}
RESUMO
Objetivou-se avaliar prevalência de cistos ovarianos em vacas leiteiras de alta produção, no pós-parto, submetidas ao regime de dieta total em três sistemas diferentes: Compost Barn, Free Stall e confinamento a céu aberto no sudoeste do estado de Goiás. Foram avaliadas 1600 vacas, HPB, de sete rebanhos, das quais $792(49,5 \%)$ estavam vazias e com atraso nos protocolos reprodutivos. Ao exame ultrassonográfico o escore ovariano foi classificado em: Grau 1 (61,4\%), Grau 2 (18,1\%), Grau $3(4,0 \%)$ e Cistos Ovarianos (16,5\%). Quanto ao sistema de produção as prevalências de cistos foram: $16 \%$ no Free Stall, 17,2\% no Compost Barn e 16,2\% no confinamento a céu aberto, diferenças somente numéricas, mas não significativas estatisticamente. $84,7 \%(\mathrm{n}=131)$ dos animais estavam em anestro, na presença do diagnóstico cisto folicular. $67,7 \%$ desses animais, situavam-se entre 60 a 180 dias pós-parto. Divididos em $27,5 \%$ entre 60 e 90 dias, $24,5 \%$ entre 91 e 120 dias, $15,5 \%$ entre 121 e 150 dias e $32,5 \%$ entre 151 e 500 . A prevalência dos cistos em função do número de lactações foi: $29,8 \%$ na primeira, $26,7 \%$ na segunda, $24,4 \%$ na terceira, $5,3 \%$ na quarta, $4,6 \%$ na quinta e $9,2 \%$ na sexta, resultados estatísticos significativos para maior ocorrência de cistos nas três primeiras lactações. Quanto ao escore de condição corporal $62,6 \%$ das vacas com cisto ovariano mostraram-se com escore entre 2,0 a 2,5, 25,2\% entre 2,6 a 3,0 e 12,2\% entre 3,1 a 4,0, resultados significativos, e a chance de vacas em balanço energético negativo desenvolverem cistos foi 5,1 vezes maior, quando comparadas com animais em escore corporal ideal. Os cistos foliculares apresentam-se com elevada casuística em vacas de alta produção leite, impactando em subfertilidade e prejuízos importantes na pecuária leiteira mundial, assim, faz-se necessário outros estudos visando o desenvolvimento de medidas preventivas mais eficazes no controle deste quadro.
\end{abstract}

PALAVRAS-CHAVE: reprodução, ovários císticos, escore corporal, bovinos.

\begin{abstract}
This study aimed to evaluate the follicular cysts prevalence in high production dairy cows, post-partum, under total diet regime in three different systems: Compost Barn, Free Stall and opened feedlot. There were evaluated 1600 cows, HPB, seven flocks, of which 792 (49.5\%) were empty and late in the reproductive protocols. The examination sonographic ovarian score was distributed as: Score $1(61.4 \%)$, Score $2(18,1)$ Score $3(4.0 \%)$ and Cystic Ovary (16.5\%). As for the production system the cysts prevalence were $16 \%$ in Free Stall, $17.2 \%$ in Compost Barn and $16.2 \%$ in opened feedlot, without significant difference. $84.7 \%(n=$ 131) of animals were in anestrus in the presence of diagnosis follicular cyst. $60.7 \%$ of animal with follicular cysts were 60 to 180 days postpartum. Divided in $27.5 \%$ between 60 and 90 days, 24.5\% between 91 and 120 days, $15.5 \%$ between 121 and 150 days and $32.5 \%$ between 151 and 500 postpartum. The cysts prevalence according to lactation number were: $29.8 \%$ in first, $26.7 \%$ in second, $24.4 \%$ in third, $5.3 \%$ in fourth, $4.6 \%$ in fifth and $9.2 \%$ in sixth, statistically significant results to higher cysts occurrence in the first three lactations. For body condition score $62.6 \%$ of animals with ovarian cyst were with scores from 2.0 to $2.5,25.2 \%$ with a score of 2.6 to 3.0 and $12.2 \%$ with score of 3.1 to 4.0 also significant results. Where the chance of cows with low body score develop cysts was 5.1 times higher compared to animals with good score. Follicular cysts present high prevalence in high producing dairy cows, impacting sub-fertility and causing important damage in global dairy farming, thus, it is necessary further studies for the development of more effective preventive measures to control this situation.
\end{abstract}

KEYWORDS: reproduction, cystics ovarians, body score, bovine. 


\section{INTRODUÇÃO}

O estado de Goiás está entre os cinco maiores produtores de leite do Brasil, como reflexo do avanço tecnológico, aumento da demanda de grãos, valorização regional das terras (IBGE 2018). Assim, a cadeia do agronegócio vem se organizando, com vistas na seleção criteriosa de vacas com alta produção em sistemas intensivos, priorizando, o bem-estar animal, a redução do custo do leite produzido e o incremento no volume diário por área (ZOCCAL \& PEREIRA 2016). A eficiência reprodutiva é alicerçada na dieta constante e de boa qualidade, que é a peça chave para o sucesso do setor, visando garantir o fornecimento e qualidade do produto ao longo de todo o ano. Desta forma, justifica-se a necessidade dos sistemas de confinamento e dieta total para animais de alto valor zootécnico destinados a produção de leite no estado (ZOCCAL \& PEREIRA 2016).

O cisto ovariano é uma das causas mais impactantes de infertilidade em vacas de alta produção (TODOROKI \& KANEKO 2006, AMER \& BADR 2007). É diagnosticado geralmente no período pós-parto, com prevalência oscilando entre rebanhos de 6 - 30\% (FERNANDES et al. 2005, SANTOS \& VASCONCELOS 2006, SANTOS et al. 2009, FAIDER et al. 2016). Ressalta-se que $48 \%$ destes cistos foram diagnosticados em vacas com média de 60 dias pós-parto e repercutiram em perdas econômicas significativas, na ordem de 230 dólares por lactação (FERNANDES et al. 2005, SANTOS et al. 2009, NASCIMENTO \& SANTOS 2010, FAIDER et al. 2016).

A formação do cisto folicular, em síntese, ocorre pela ausência de ovulação e permanência do folículo dominante, com diâmetro superior a $20 \mathrm{~mm}$ mantido por período de 10 dias na ausência de corpo lúteo (MADUREIRA et al. 2002, SILVIA et al. 2002, FAIDER et al. 2016), mas este diagnóstico deve ser criterioso, pois vacas leiteiras de alta produção, naturalmente chegam a ovular folículos grandes com 16 a $18 \mathrm{~mm}$ (GÜMEN et al. 2003, GÜMEN \& WILTBANK 2005, VANHOLDER et al. 2006, SANTOS et al. 2009).

O mecanismo de desenvolvimento dos cistos ovarianos em ruminantes ainda não se encontra completamente elucidado, várias hipóteses são conjecturadas, como a síntese reduzida do hormônio liberador de gonadotropinas $(\mathrm{GnRH})$, em associação com limitado pico pré-ovulatório do hormônio luteinizante (LH), ou ainda, a resposta insuficiente do estrógeno na retroalimentação e pulsatilidade dos hormônios do eixo hipotámo-hipofisário (RIBADU et al. 2000, VANHOLDER et al. 2006, SANTOS et al. 2009).

$O$ estresse também favorece o desenvolvimento dos cistos, pois o cortisol endógeno suprime a onda pré-ovulatória de LH, a partir da inibição dos estímulos do GnRH (SANTOS et al. 2009, FAIDER et al. 2016).

O presente estudo teve como objetivo avaliar prevalência de cistos foliculares em vacas leiteiras de alta produção, no pós-parto, submetidas ao regime de dieta total em três sistemas diferentes Compost Barn, Free Stall e confinamento a céu aberto.

\section{MATERIAL E MÉTODOS}

Foram avaliados por exames ginecológicos, palpação retal e ultrassonografia 1600 vacas, raça holandesa (HPB), com 60 - 500 dias em lactação (DEL), das quais, 792 (49,5\%) estavam vazias e fora do período de espera voluntário, portanto, identificadas em atraso reprodutivo ou subfértis nos sistemas de gerenciamento dos rebanhos que integraram este estudo.

Os animais foram provenientes de sete propriedades leiteiras, com produção média de $24 \mathrm{~kg}$ de leite, entre a primeira e sexta lactações, confinadas em sistemas de Free-Stall, Compost Barn ou Semiconfinamento, sob manejo de três ordenhas diárias e alimentação com dieta total. As propriedades situavamse no sudoeste do estado de Goiás. Todas as vacas amostradas neste experimento, também tiveram a identificação do escore de condição corporal (1 a 5), que foram correlacionados com os dados referentes ao comportamento reprodutivo, número de lactações, escore ovariano e dias após o parto, conforme metodologias padronizadas em estudos semelhantes (MADUREIRA et al. 2002, SANTOS et al. 2009, FERREIRA et al. 2010).

Rebanho 1 - Sistema Compost Barn com cama de maravalha, 322 animais em lactação, produção média de $28 \mathrm{~kg} / \mathrm{leite}$, dieta à base de silagem de milho, feno de tifton, casca de soja, gordura protegida, farelo de soja, minerais e vitaminas.

Rebanho 2 - Sistema Free-Stall com cama de areia, 318 animais em lactação, produção média de 30 $\mathrm{kg} / \mathrm{leite}$, dieta à base de silagem de milho, silagem grão úmido de milho, feno de tifton, casca de soja, gordura protegida, farelo de soja, minerais e vitaminas.

Rebanho 3 - Sistema Compost Barn com de cama de maravalha, 240 animais em lactação, produção média de $34 \mathrm{~kg} / \mathrm{leite}$, dieta à base de silagem de milho/sorgo, feno de tifton, casca de soja peletizada, polpa cítrica, gordura protegida, farelo de soja, milho moído, minerais e vitaminas.

Rebanho 4 - Sistema de confinamento a céu aberto, com 270 animais em lactação, produção média de 
$25 \mathrm{~kg} / \mathrm{leite}$, dieta à base de silagem de milho, feno de tifton, milho moído, farelo de Soja, minerais e vitaminas.

Rebanho 5 - Sistema de confinamento a céu aberto, com 150 animais em lactação, produção média de $23 \mathrm{~kg} / \mathrm{leite}$, dieta à base de silagem de milho, feno de tifton, milho moído, farelo de soja minerais e vitaminas.

Rebanho 6 - Sistema de confinamento a céu aberto, com 170 animais, produção média de $25 \mathrm{~kg} / \mathrm{leite}$, dieta à base de silagem de milho, milho moído, feno de tifton, farelo de soja, casca de soja peletizada, polpa cítrica, minerais e vitaminas.

Rebanho 7 - Sistema Free-Stall cama de borracha, com 130 animais, produção média de 25kg/leite, dieta à base de silagem de milho, milho moído, feno de tifton, farelo de soja, casca de soja, minerais e vitaminas.

As 792 vacas diagnosticadas como vazias, tiveram os ovários classificados em escores:

Grau 1 - Vacas ciclando, com ovários medindo acima de $30 \mathrm{~mm}$, macios, presença de corpo lúteo (CL), ou útero com turgidez acentuada, sugerindo a presença de folículos grandes $(10 \mathrm{~mm})$ estrógeno-ativos.

Grau 2 - Vacas sem CL, porém, com presença de estruturas com comprimento entre $15-30 \mathrm{~mm}$, turgidez no útero. Nesta categoria também foram incluídas fêmeas com folículos com potencial para atingirem a fase de dominância (> 8,5 mm), mas não ovulam.

Grau 3 - Vacas sem CL, com ovários pequenos, duros e lisos. Nesta categoria também foram inclusas fêmeas com folículos menores que $8 \mathrm{~mm}$ de diâmetro no ovário as quais não teriam condição de chegar até a divergência (MADUREIRA et al. 2002).

Cisto Ovariano - Vacas com a presença de estruturas ovarianas maiores que $20 \mathrm{~mm}$ de diâmetro, mensuradas com auxílio do equipamento de ultrassonografia (SIUI-3300) com probe linear retal e frequência de $7,5 \mathrm{mhz}$, o mesmo técnico realizou todas as medidas referentes ao estudo, que foi conduzido entre os anos de 2015 a 2017.

Os animais com ovários Grau 1 e 2 foram considerados cíclicos, enquanto o Grau 3 e as vacas com cistos foram consideradas acíclicas, ressalta-se que a identificação de ovários císticos, é uma modificação da técnica, ora não está inclusa nas revisões que trabalharam com escores ovarianos (MADUREIRA et al. 2002, SANTOS et al. 2009), cuja descrição para cistos, é a presença de folículos maiores que $20 \mathrm{~mm}$, na ausência de corpo lúteo e que persistem por mais de 10 dias, mas não classificados como um grau dentro do escore ovariano.

$\mathrm{Na}$ análise estatística, as amostras foram obtidas por conveniência, embasada na rotina de propriedades assistidas tecnicamente por profissional autônomo, ao longo de um ano, utilizou-se o teste de 2 a 5\% de probabilidade na comparação das variáveis relativas à ocorrência de cisto nas diferentes condições. As médias de escores corporal e ovariano, assim como a média do diâmetro dos cistos foi comparada pelo teste estatístico de Tuckey e determinação do Odds Ratio (OR) ou risco relativo de desenvolvimento dos cistos em função dos dias após o parto, escore de condição corporal e número de lactações (TRIOLA 2005).

\section{RESULTADOS E DISCUSSÃO}

Os resultados referentes a prevalência de cisto ovariano em animais com diferentes lactações, classificação ovariana de vacas pós-parto, ocorrência de cistos ovarianos de acordo com o escore de condição corporal e respectivas correlações paramétricas estão sumariados nas Tabelas 1, 2, 3 e 4.

\section{Prevalência de Cisto ovariano e classificação ovariana de vacas pós-parto.}

Dentre as 792 vacas diagnosticadas como vazias observou-se, respectivamente, as seguintes classificações ovarianas: Grau 1 (61,4\%), Grau 2 (18,1\%), Grau $3(4,0 \%)$ e ovários císticos $(16,5 \%$.) Estes resultados sinalizam, que vacas de alta produção, identificadas como vazias, apresentavam-se cíclicas e com boas condições ovarianas (Tabela 1), provavelmente, mesmo nos rebanhos com maiores níveis tecnológicos, estão ocorrendo falhas significativas na observação de cio, assim como foi revisto por outros pesquisadores (MADUREIRA et al. 2002, SANTOS et al. 2009, JEENGAR et al. 2014)

A prevalência dos cistos ovarianos em vacas de leite de alta produção tem sido exaustivamente estudada na última década, e convencionalmente varia entre seis a 18\%, (TODOROKI \& KANEKO 2006, SANTOS et al. 2009, FAIDER et al. 2016), em acordo este trabalho diagnosticou $16,5 \%(n=792)$ vacas com ovários císticos, distribuídos em $59,6 \%(n=131)$ no ovário direito, $33,6 \%(n=131)$ esquerdo e $6,8 \%(n=131)$ dos casos eram cistos bilaterais, resultados estes que foram significativos estatisticamente.

Quanto ao sistema de produção a prevalência foi: 16\% em animais criados em sistema de Free Stall, $17,2 \%$ no Compost Barn e 16,2\% em confinamento a céu aberto, embora tenham ocorrido diferenças numéricas não foram significativas do ponto de vista estatístico. Os resultados assemelham-se aos apresentados LOPÉZ-GATIUS et al. (2002) e GÜMEN et al. (2003) que relataram incidência entre sete a 19\% em vacas de leite confinadas em sistema de Free Stall. 
Nos EUA, estima-se que 15\% das vacas de leite de alta produção apresentam cisto ovariano único ou formações múltiplas durante a lactação, com taxas de cura espontânea que podem ultrapassar $40 \%$, mas com atrasos preocupantes nos índices reprodutivos das propriedades (SANTOS \& VASCONCELOS 2006, CRANE et al. 2006, SANTOS et al. 2009), ainda considera, pouco frequente os quadros de cios curtos, irregulares e comportamento "ninfomaníaco", conforme eram descritos nos primeiros relatos de diagnósticos de cistos foliculares (SILVIA et al. 2002).

Esta situação também se repetiu neste estudo, já que os animais com diagnóstico de cisto ovariano não se encontravam com características de estro durante a avaliação ultrassonográfica, assim, $84,7 \%$ $(n=131)$ dos animais deste experimento, estavam em anestro na presença do diagnóstico de cisto ovariano. FERREIRA (2010) reportou que 62 a $80 \%$ das vacas de alta produção, diagnosticadas com o cisto, também se encontravam nas mesmas condições sem manifestação comportamental de estros intercorrentes.

Tabela 1. Prevalência de cisto, classificação ovariana e distribuição dos cistos entre ovário direito, ovário esquerdo e cistos bilaterais*.

Table 1. Cyst prevalence, ovarian classification and cysts distribution between right ovary, left ovary and bilateral cysts*.

\begin{tabular}{|c|c|c|c|c|c|c|c|c|}
\hline \multirow[t]{2}{*}{$\begin{array}{c}\text { Classificação } \\
\text { Ovariana }\end{array}$} & \multirow[t]{2}{*}{$\mathrm{N}$} & \multirow[t]{2}{*}{$\%$} & \multicolumn{2}{|c|}{ Ovário direito } & \multicolumn{2}{|c|}{$\begin{array}{c}\text { Ovário } \\
\text { Esquerdo }\end{array}$} & \multicolumn{2}{|c|}{$\begin{array}{c}\text { Cistos } \\
\text { Bilaterais }\end{array}$} \\
\hline & & & $\mathrm{N}$ & $\%$ & $\mathrm{~N}$ & $\%$ & $\mathrm{~N}$ & $\%$ \\
\hline Grau 1 & $486^{a}$ & $61,4^{a}$ & - & - & - & - & - & - \\
\hline Grau 2 & $143^{b}$ & $18,1^{\mathrm{b}}$ & - & - & - & - & - & - \\
\hline Grau 3 & $32^{c}$ & $4,0^{c}$ & - & - & - & - & - & - \\
\hline Ovário Cístico & $131^{b}$ & $16,5^{\mathrm{b}}$ & 78 & $59,6^{a}$ & 44 & $33,6^{b}$ & 9 & $6,8^{c}$ \\
\hline Total & 792 & 100 & 131 & 100 & 131 & 100 & 131 & - \\
\hline
\end{tabular}

*Letras minúsculas diferentes nas mesmas linhas ou colunas diferem-se entre si pelo teste de $x 2(p<0,05)$.

O presente estudo, balizou-se também, na presença de estruturas ovarianas $\geq 20 \mathrm{~mm}$, para o diagnóstico dos cistos e ainda distribui os cistos conforme sua localização, no ovário direito foram identificados $59,6 \%(n=131)$, no ovário esquerdo $33,6 \%(n=131)$ e a disposição bilateral em $6,8 \%(n=131)$, resultados estatisticamente significativos, de acordo com a Tabela 2, que está em consonância com outros pesquisadores, cujos trabalhos foram pautados na distribuição dos cistos foliculares em vacas de leite (ZULU \& PENNY 1998, MADUREIRA et al. 2002, HATLER et al. 2003, PATTON et al. 2007). Assim, o diâmetro das estruturas verificadas variou de 20 a $50 \mathrm{~mm}$ (mediana $24 \mathrm{~mm}$ ), estas medidas são semelhantes as observadas por FERNANDES et al. (2005) e SANTOS et al. (2009) em trabalho com cistos em vacas de alta produção. A Tabela 2 apresenta a descrição sintetizada das medidas dos diâmetros dos cistos foliculares diagnosticados nas vacas deste estudo.

Tabela 2. Descrição dos cistos ovarianos de acordo com valor mínimo, percentil 25, mediana, percentil 75 e desvios padrões, estruturas mensuradas em milímetros com auxílio de ultrassonografia com probe linear retal e frequência de $7,5 \mathrm{mhz}$. $^{*}$

Table 2. Description of ovarian cysts according to minimum value, percentile 25, median, $75 \mathrm{~h}$ percentile and standard deviations, structures measured in millimeters using ultrasound with rectal linear probe and frequency of $7.5 \mathrm{mhz}$.

\begin{tabular}{cccccccc}
\hline Cistos & Mín. & P25 & Med. & Média & P75 & Máx. & $\begin{array}{c}\text { Desvio } \\
\text { Padrão }\end{array}$ \\
\hline $\begin{array}{c}\text { Medidas } \\
(\mathrm{mm})\end{array}$ & 20 & 22 & 24 & 25,4 & 29 & 50 & 5,38 \\
\hline
\end{tabular}

${ }^{*}$ mín = mínimo, P25 = percentil 25, P75 = percentil 75, Med. = mediana, Max = máximo, mm = milímetro.

\section{Prevalência de cisto ovariano de acordo com os dias em lactação e número de partos.}

Este estudo contemplou o diagnóstico de cistos ovarianos em vacas holandesas com lactação entre 60 a 500 dias pós-parto, que foi realizado pela avaliação ultrassonográfica, em conformidade com VASCONCELOS et al. (2009).

Em consonância com outros trabalhos realizados em diferentes países e sistemas de produção com dieta total (ZULU \& PENNY 1998, FERNANDES et al. 2005, GAEBLER et al. 2015), o presente estudo 
demonstrou que $80,6 \%$ dos animais com diagnóstico de cisto ovariano estavam entre 60 e 180 dias pós parto, assim conforme a seguintes distribuição $27,6 \%$ entre 60 e 90 dias, 24,6\% entre 91 a 120 dias, 15,5\% entre 121 a 150 dias, $12,9 \%$ entre 151 a 180 dias, $13,3 \%$ entre 181 a 210 dias e $6,1 \%>210$ dias após o parto, com associação estatística entre a ocorrência dos cistos e o período pós-parto, com OR (odds ratio) indicando o risco 3,8 vezes maior para o desenvolvimento de cistos em animais até 180 dias após o parto.

Mais de $70 \%$ dos cistos ovarianos ocorrem entre 16 a 50 dias após o parto, e a maior ocorrência distribui-se entre 30 a 40 dias (GAEBLER et al. 2015, TANEMURA et al. 2016). Já ZULU \& PENNY (1998) descreveram a ocorrência de cistos ovarianos em vacas de leite de 20 a 150 dias após o parto. A presença de cistos alonga o intervalo entre partos, refletindo diretamente no aumento de 22 a 64 dias no período de serviço (FERNADES et al. 2005). PATTON et al. (2007) relataram que vacas com cistos e vacas normais apresentaram intervalo parto-primeira ovulação de 58 e 12 dias, respectivamente.

Os principais fatores atribuídos a maior ocorrência dos cistos ovarianos neste período, pico de lactação, em que o animal entra em fase de balanço energético negativo, por vezes subclínico, segundo TANEMURA et al. 2016, representa um fator predisponente à ocorrência de cistos. Além disso, é considerado o período de maior estresse durante a lactação (FERNANDES et al. 2005, NELSON et al. 2010).

DOBSON et al. (2000) e GAEBLER et al. ( 2015) citam que, em condições de concentrações séricas de cortisol elevadas, ocorrem alterações significativas na dinâmica folicular, principalmente por efeitos na liberação de LH. Na fase inicial da lactação ocorre um progressivo aumento na ingestão de alimentos que resulta em maior fluxo sanguíneo hepático, ocasionando aumento no metabolismo da progesterona produzida pelo tecido luteal de um corpo lúteo normal e, posteriormente, redução na concentração circulante (VASCONCELOS et al. 2003, SANTOS et al. 2009)

Já a prevalência dos cistos baseados em número de lactações foi, respectivamente, $29,8 \%(n=131)$ na primeira, $26,7 \%(n=131)$ na segunda, $24,4 \%(n=131)$ na terceira, $5,3 \%(n=131)$ na quarta, $4,6 \%$ $(n=131)$ na quinta e $9,2 \%(n=131)$ na sexta, resultados significativos estatisticamente para maior ocorrência de cistos nas três primeiras lactações nas condições amostrais deste estudo (Tabela 3).

O número de lactações discordou dos resultados obtidos por FERNANDES et al. (2005), em que foi discutida maior prevalência de cistos ovarianos em animais mais velhos a partir do quinto parto com 15,46\% de prevalência, enquanto, primíparas e vacas de segundo parto demonstraram somente $6,13 \%$ de prevalência de cistos foliculares. Este resultado pode ser em função da elevada taxa de descarte de vacas nas propriedades amostradas a partir da quinta lactação.

Tabela 3. Prevalência de Cisto ovariano em respectivas lactações*.

Table 3. Ovarian cyst prevalence in respective lactations*.

\begin{tabular}{|c|c|c|c|c|}
\hline Lactações & $\begin{array}{l}\text { Número de animais } \\
\text { examinados por } \\
\text { lactação }\end{array}$ & $\begin{array}{l}\text { Prevalência de vacas } \\
\text { com atraso } \\
\text { reprodutivo por } \\
\text { lactação }(\%)\end{array}$ & $\begin{array}{l}\text { Diagnóstico de } \\
\text { cisto por } \\
\text { lactação }\end{array}$ & $\begin{array}{c}\text { Prevalência de } \\
\text { cistos ovarianos } \\
\text { por categoria de } \\
\text { lactação }(\%)\end{array}$ \\
\hline 1a e 2 $2^{\underline{a}}$ Lactações & $555^{a}$ & $70,1 \%^{a}$ & $74^{a}$ & $56,5 \%^{a}$ \\
\hline $3^{a}$ e e $4^{a}$ Lactações & $179^{b}$ & $22,6 \%{ }^{b}$ & $39^{b}$ & $29,8 \%$ b \\
\hline $5^{\mathrm{a}}$ e 6 Lactações $^{* *}$ & $58^{c}$ & $7,3 \% c$ & $18^{c}$ & $13,7 \%^{\mathrm{c}}$ \\
\hline Total(n) & 792 & $100,0 \%$ & 131 & $100,0 \%$ \\
\hline
\end{tabular}

*Letras minúsculas diferentes na mesma coluna, diferem entre si pelo teste de $x 2(p<0,05)$.

** O número de animais nesta categoria foi inferior em decorrência do manejo das propriedades amostradas que realizam descarte voluntário de animais a partir da quarta lactação.

\section{Ocorrência de Cistos Ovarianos de acordo com o escore de condição corporal}

Das 131 vacas diagnosticadas com cistos ovarianos $62,6 \%$ estavam com escore entre 2,0 a $2,5,25,2 \%$ com escore 2,6 a 3,0 e 12,2\% escore 3,1 a 4,0, com resultados estatísticos significativos, com OR (Odds ratio) 5,1 vezes maior para o desenvolvimento de cistos nos animais com baixo escore corporal.

Quanto à disposição dos cistos ovarianos conforme o Escore de condição corporal dos animais (ECC). O ECC é uma medida visual, prática e de baixo custo (BARTOLOME et al. 2005), usada para monitorar o estado nutricional e o desempenho reprodutivo de vacas de leite em várias regiões do mundo, pois é determinante na reconcepção e na produtividade geral do rebanho (FERNANDES et al. 2005, TANEMURA et al. 2016).

O manejo nutricional ajustado proporciona a condição corporal desejável nos diferentes estágios de produção, cujas exigências aumentam no terço final da gestação e no intervalo de parição (início da lactação) 
até a próxima concepção (pico da lactação), segundo SANTOS et al. (2009). A avaliação do escore de condição corporal permite a análise das práticas de manejo adotadas e pode subsidiar aos produtores dados para melhoria na eficiência dos programas de manejo reprodutivo e nutricional (BARTOLOME et al. 2005).

De acordo com FAIDER et al. (2016) e TANEMURA et al. (2016), as mudanças no ECC entre o final da gestação, parto e início da lactação têm sido responsáveis por diminuir o desempenho produtivo e reprodutivo pós-parto e aumentar a incidência de transtornos reprodutivos em vacas de leite.

Tabela 4. Relação entre escore corporal e cistos ovarianos*.

Table 4. Relationship between body score and ovarian cysts*.

\begin{tabular}{cccc}
\hline Escore Corporal & Animais classificados & Diagnóstico de cisto & $\begin{array}{c}\text { Ocorrência/Escore } \\
\text { corporal }(\%)\end{array}$ \\
\hline $2,0-2,5$ & $540^{\mathrm{a}}$ & $82^{\mathrm{a}}$ & $62,6 \%^{\mathrm{a}}$ \\
$2,6-3,0$ & $181^{\mathrm{b}}$ & $33^{\mathrm{b}}$ & $25,2 \% \%^{\mathrm{b}}$ \\
$3,1-4,0$ & $71^{\mathrm{c}}$ & $16^{\mathrm{c}}$ & $12,2 \% \%^{\mathrm{c}}$ \\
\hline Total & 792 & 131 & $100 \%$ \\
\hline
\end{tabular}

*Letras minúsculas diferentes na mesma coluna, diferem-se entre si pelo teste de $\mathrm{x} 2(\mathrm{p}<0,05)$.

O número de cistos ovarianos em animais com escore da condição corporal inferior ou superior foi maior quando comparados com aqueles de escore intermediário (Tabela 4). ROCHE et al. (2000) citaram relação inversa entre escore de condição corporal e incidência de cistos. A casuística de ocorrência de cistos foi maior entre animais com menor escore corporal do que aqueles considerados em escore ideal, visto que, $70 \%$ dos animais estavam abaixo de $(2,75)$ e $30 \%$ acima de $(3,0)$, justificando inversão da relação causaefeito. Enfatiza-se que condição corporal ruim pode favorecer o aumento na ocorrência de cistos, mas, a mudança nos níveis de hormônios basais em animais com cistos pode contribuir para alteração do escore de condição corporal. Animais com cistos ovarianos apresentam redução de fertilidade por prejuízos causados a concepção pela presença do cisto (VASCONCELOS et al. 2003, SANTOS et al. 2009, FAIDER et al. 2016, TANEMURA et al. 2016).

Em síntese, inúmeros fatores estão relacionados com o maior risco de desenvolvimento de cistos ovarianos, como perda de escore de condição corporal, época do ano e desordens metabólicas no pós-parto, número de lactações (LÓPEZ-GATIUS et al. 2002, MADUREIRA et al. 2002, VASCONCELOS et al. 2009, GAEBLER et al. 2015, FAIDER et al. 2016).

Exaustivamente discute-se o desenvolvimento do cisto, a partir, de desequilíbrios endócrinos no eixo hipotálamo-hipófise-gônadas (SANTOS et al. 2009, GAEBLER et al. 2015). GÜMEN et al. (2003) e GÜMEN \& WILTBANK (2005) relataram que a formação de um folículo ovariano grande, similar a um cisto folicular, pode ser induzida pela aplicação de estradiol, que induz o pico de $\mathrm{GnRH}$ e $\mathrm{LH}$, na ausência da luteinização subsequente, seja por aplicação de estradiol na ausência de um folículo dominante (NELSON et al. 2010) ou por remoção do corpo hemorrágico (GÜMEN \&WILTBANK 2005). Mas, fatores predisponentes de origens múltiplas devem ser aventados como fatores predisponentes para o desenvolvimento desta síndrome, conforme exposto neste estudo.

\section{CONCLUSÃO}

Os cistos foliculares apresentam-se com elevada casuística em vacas de alta produção leite, impactando em subfertilidade e prejuízos importantes na pecuária leiteira mundial, assim, fazem-se necessários outros estudos visando o desenvolvimento de medidas preventivas mais eficazes no controle desta síndrome. A sintomatologia clínica observada com maior frequência foi o anestro, assim como, as vacas listadas como subférteis neste experimento encontravam-se cíclicas, confirmando a ineficácia dos sistemas de observação de cio em rebanhos altamente tecnificados.

\section{REFERÊNCIAS}

AMER H \& MAHDI AB 2008. Hormonal profiles associated with treatment of cystic ovarian disease with GnRH and PGF2 $\alpha$ with and without CIDR in dairy cows. Livestock Research for Rural Development 20: 51-56.

BARTOLOME JA et al. 2005. Strategies for the diagnosis and treatment of ovarian cysts in dairy cattle. Journal of the American Veterinary Medical Association 277: 1409-1414.

CRANE MB et al. 2006. Association between milk production and treatment response of ovarian cysts in lactating dairy cows using the Ovsynch protocol. Theriogenology 66: 1243-1248.

DOBSON H et al. 2000. Ultrasonography and hormone profiles of adrenocorticotrophic hormone (ACTH) induced persistent ovarian follicles (cysts) in cattle. Journal of Reproduction and Fertility 120: 405-410. 
FERNANDES CAC et al. 2005. Fatores predisponentes para cistos ovarianos em vacas da raça holandesa. Ars Veterinaria 21: 287-295.

FERREIRA AM. 2010. Reprodução da fêmea bovina: fisiologia aplicada e problemas mais comuns (causas e tratamentos). 1.ed. Juiz de Fora: Editar. 420p.

GAEBLER E et al. 2015. Fate of follicular ovarian cysts in early postpartum dairy cows treated with PRID/PGF or PRID/PGF plus eCG. Tierarztl Prax Ausg G Grosstiere Nutztiere 43: 331-339.

GÜMEN A et al. 2003. Follicular size and response to Ovsynch versus detection of estrus in anovular and ovular lactating dairy cows. Journal of Dairy Science 86: 3184-3194.

GÜMEN A \& WILTBANK MC. 2005. Follicular cysts occur after a normal estradiol-induced GnRH/LH surge if the corpus hemorrhagicum is removed. Reproduction 129: 737-745.

HATLER TB et al. 2003. Relationship between endogenous progesterone and follicular dynamics in lactating dairy cows with ovarian follicular cysts. Biology of Reproduction 69: 218-223.

IBGE. 2018. Instituto Brasileiro de Geografia e Estatística. Disponível em: http://www.ibge.gov.br. Acesso em: 15 fev. 2019.

JEENGAR $\mathrm{K}$ et al. 2014. Ovarian cysts in dairy cows: old and new concepts for definition, diagnosis and therapy. Animal Reproduction 11: 63-73.

LÓPEZ-GATIUS F et al. 2002. Risk factors for postpartum ovarian cysts and their spontaneous recovery or persistence in lactating dairy cows. Theriogenology 58: 1623-1632.

MADUREIRA EH et al. 2002. Sincronização com progestágenos - Biotecnologia da reprodução em bovinos. In: 1 Simpósio Internacional de Reprodução Animal aplicada. Resumos... Londrina. p.117-128.

NASCIMENTO FE \& SANTOS LR. 2010. Patologia dareprodução dos animais domésticos. 3.ed. Rio de Janeiro: Guanabara Koogan.

NELSON ST et al. 2010. Risk factors associated with cystic ovarian disease in Norwegian dairy cattle. Acta Veterinaria Scandinavica 43: 52-60.

PATTON J et al. 2007. Relationships among milk production, energy balance, plasma analytes, and reproduction in Holstein-Friesian cows. Journal of Dairy Science 90: 649-658.

RIBADU AY et al. 2000. The role of LH pulse frequency in ACTH-induced ovarian follicular cysts in heifers. Animal Reproduction Science 64: 21-31.

ROCHE, JF et al. 2000. Resumption of reproductive activity in the early postpartum period of cows. In: Congresso Della Societa Italiana di Buiatria, Aiago. Proceedings... 32: 45-49.

SANTOS RM \& VASCONCELOS JLM. 2006. Ingestão de concentrado e concentração plasmática de progesterona em vacas da raça Holandesa. Arquivo Brasileiro de Medicina Veterinária e Zootecnia 58: 1162-1167.

SANTOS RM et al. 2009. Cisto ovariano em vacas de leite: incidência, resposta à aplicação de GnRH e desempenho reprodutivo. Arquivo Brasileiro de Medicina Veterinária e Zootecnia 61: 527-532.

SILVIA WJ et al. 2002. Ovarian follicular cysts in dairy cows: an abnormality in folliculogenesis. Domestic Animal Endocrinology 23: 167-177.

TANEMURA K et al. 2016. Development of ovarian diseases in dairy cows with a history of fatty liver, and their prognosis. Journal of Veterinary Medical Science 78: 755-760.

TRIOLA MF. 2005. Introdução à estatística. 9.ed. Rio de Janeiro: LTC. 656p.

TODOROKI J \& KANEKO H. 2006. Formation of follicular cysts in cattle and therapeutic effects of controlled internal drug release. Journal of Reproduction and Development 52: 1-11.

VASCONCELOS JL. et al. 2003. Acute reduction in serum progesterone concentrations after feed intake in dairy cows. Theriogenology 60: 795-807.

VANHOLDER T et al. 2006. Aetiology and pathogenesis of cystic ovarian follicles in dairy cattle: a review. Reproduction Nutrition Development 46: 105-119.

VILLADIEGO FAC et al. 2016. Parâmetros reprodutivos e produtivos em vacas leiteiras de manejo free stall. Pesquisa Veterinária Brasileira 36: 55-61.

ZOCCAL R \& PEREIRA VF. 2016. Panorama do Leite. Juiz de Fora: Embrapa Gado de Leite. 12p. (Boletim eletrônico mensal 8).

ZULU VC \& PENNY C. 1998. Risk factor of cystic ovarian disease in dairy cattle. Journal of Reproduction and Development 44: 191-195. 